\title{
Editorial: Understanding Coexistence With Wildlife
}

\author{
Simon Pooley ${ }^{1,2 *}$, John D. C. Linnell ${ }^{3,4}$, Ursula Münster ${ }^{5}$, Thom van Dooren ${ }^{5,6}$ and \\ Alexandra Zimmermann ${ }^{7}$ \\ 1 Department of Geography, Birkbeck, University of London, London, United Kingdom, ${ }^{2}$ School of Life Sciences, University of \\ KwaZulu-Natal, Scottsville, South Africa, ${ }^{3}$ Norwegian Institute for Nature Research (NINA), Trondheim, Norway, ${ }^{4}$ Inland \\ Norway University of Applied Sciences, Department of Forestry and Wildlife Management, Koppang, Norway, ${ }^{5}$ Faculty of \\ Humanities, Oslo School of Environmental Humanities, University of Oslo, Oslo, Norway, ${ }^{6}$ Faculty of Arts and Social \\ Sciences, The University of Sydney, Sydney, NSW, Australia, ${ }^{7}$ Wildlife Conservation Research Unit, Department of Zoology, \\ Mathematical, Physical and Life Sciences Division, University of Oxford, Oxford, United Kingdom
}

Keywords: human-wildlife coexistence, human-wildlife conflict (HWC), diversity, interdisciplinary, conservation management

\section{Editorial on the Research Topic}

Understanding Coexistence With Wildlife

\section{OPEN ACCESS}

Edited by: Krithi K. Karanth

Centre for Wildlife Studies, India

Reviewed by:

Jared D. Margulies,

University of Alabama, United States

Adrian Treves,

University of Wisconsin-Madison,

United States

*Correspondence:

Simon Pooley

s.pooley@bbk.ac.uk

Specialty section:

This article was submitted to

Human-Wildlife Dynamics,

a section of the journal

Frontiers in Conservation Science

Received: 07 December 2021

Accepted: 10 January 2022

Published: 14 February 2022

Citation:

Pooley S, Linnell JDC, Münster U, van Dooren T and Zimmermann A (2022)

Editorial: Understanding Coexistence With Wildlife.

Front. Conserv. Sci. 3:830971. doi: 10.3389/fcosc.2022.830971

\section{INTRODUCTION}

As humans and wildlife come into increasing contact under the pressures of climate change, human development, successful conservation and wildlife recovery, and zoonoses, it is urgent that we learn to facilitate coexistence with wildlife in shared multi-use landscapes, for the wellbeing of both wildlife and people. The terms "human-wildlife conflict" and "human-wildlife coexistence" are both used in work aiming to achieve this, but in both cases a variety of definitions exist. While the term "coexistence" is being increasingly mentioned, possibly linked to a preference for a positive framing of human-wildlife interactions in particular, it is not often defined (see however Pooley in this special issue), and remains understudied. This is partly because conservation scientists are less familiar and less comfortable with the kinds of questions and methodologies required to study human-wildlife coexistence. It is also easier to study things you can count (impacts, e.g., attacks, extent of damage or frequency of interactions) than coexistence, which often involves not doing things (e.g., refraining from retaliation or protesting). This collection of papers offers the most comprehensive and cross disciplinary examination of human-wildlife coexistence published so far.

Human-wildlife conflict research increasingly draws on approaches from a diversity of social science and humanities disciplines in order to better understand human-human conflicts over interactions with wildlife. The emphasis in human-wildlife conflict is on understanding and addressing conflicts between different groups of people over wildlife, and reducing negative impacts of wildlife on humans and vice versa. Here, research often focuses on risks and benefits of sharing a landscape with wild animals of conservation concern, and attempts to analyse and influence decision-making over how to do so. Solutions often proposed include separating humans and wildlife, or providing material benefits and compensations to those sharing landscapes with wildlife. This is vital work of direct relevance to policymakers and managers. Some additional dimensions that human-wildlife coexistence studies add to this research focus include a direct interest in positive human-wildlife interactions, and in this context, broader consideration of different ways of valuing and interacting with wildlife and the natural world. 
In our call for papers for this special issue, we asked contributors to think, about what the scope of human-wildlife coexistence should encompass, and how to study it. We wanted to learn more about coexistence from those places where it is being actively cultivated and researched. The focus of this special issue is on reasons for-and approaches to-coexistence which are not directly related to the material costs or benefits of living with particular species of wild animals. We were particularly interested in human-wildlife interactions in "everyday" shared/mixed-use landscapes, rather than only iconic conservation landscapes.

We did not offer contributors any definition of coexistence; rather, we suggested that authors should think through their own conceptions of coexistence. We suggest that conservationists should take care when generalizing such conceptions when attempting to facilitate coexistence in particular scenarios of human-wildlife interaction. We agree with contributors Glikman et al. when they advocate for working with those with relevant interests to define coexistence for particular scenarios. As noted by Pooley in his perspective piece, this requires self-reflexivity and recognition of difference.

\section{DIVERSITY IN HUMAN-WILDLIFE COEXISTENCE}

This special issue offers a rich diversity of perspectives on, and approaches to, human-wildlife coexistence-without claiming to represent that diversity comprehensively. We were delighted to received submissions from authors with backgrounds from both the Global North and South. Contributors come from a diversity of academic and sectoral backgrounds, with training variously in applied sciences, natural and social sciences, including anthropology, biology, conservation science, critical social science, environmental science, forestry, geography and zoology. Several papers are interdisciplinary efforts. The geographic range of the studies is also reasonably wide, spanning North America, Europe, and South Asia.

Although we collectively selected those abstracts that fitted our aims for the special issue, and checked first submissions to confirm their fit, we did not edit every paper (not appropriate for any we authored or co-authored, for instance). We are pleased with the stimulating diversity of approaches and proposals included, but equally these do not necessarily represent our own views or approaches.

\section{ORGANIZATION OF THE MATERIALS}

We have presented the shorter opinion and perspective pieces first (Part 1), followed by the longer research papers (Part 2 ). The former raise key conceptual matters influencing how we think about human-wildlife coexistence. These include reflections on whether and how to define human-wildlife coexistence and some of the key ethical implications of trying to facilitate coexistence (Pooley), negative and positive dimensions of coexistence and how to encourage the latter (Bhatia), the importance of not excluding conflict from conceptions of coexistence (Hill), and the usefulness of relational rather than dualistic frameworks for thinking about human-wildlife interactions (Schroer). Glikman et al.'s surveys reveal the diversity of perspectives among conservationists on concepts of coexistence, tolerance and acceptance. Kaltenborn and Linnell explore how coexistence ideas fit with the many different conservation subdisciplines, strategies and paradigms currently competing for primacy.

The richness of the discussions and investigations in the full-length research papers (Part 2) are too diverse to summarize here, so a few general points must suffice. Notably, the selected papers encompass studies of a wide range of those with important interests in human-wildlife coexistence scenarios, including: conservation managers (Vance Martin et al.), ranchers (Bogezi et al.), farmers (Thinley et al.), and locals including villagers sharing landscapes with wildlife (Toncheva and Fletcher; Thekaekara et al.). This demonstrates the need to consider a wide range of interests-not forgetting those of wild animals-when attempting to understand and foster coexistence.

The historical and cultural dimensions required to make sense of the dynamic nature of human-wildlife relations over time are the focus of papers by Broz et al., Oommen, and Thekaekara et al. Papers by Oommen, Nair et al., and Thekaekara et al. emphasize what we can learn from indigenous approaches to coexisting with wild animals that can have negative impacts on humans, their crops or livestock. Broz et al. provide insights into the emerging discourse of veterinization associated with zoonoses and wildlife disease management.

Finally, while we do not advocate for any one approach to fostering human-wildlife coexistence, several papers in this special issue offer fascinating recommendations for doing so, including conceptual frameworks suggested by Pettersson et al., and Toncheva and Fletcher.

\section{CONCLUSION}

Thinking about human-wildlife coexistence requires us to widen the aperture on what we consider important in the study of human-wildlife interactions, and therefore on how to study them. This special issue will introduce readers to ideas and approaches and readings not often encountered in mainstream conservation science contexts, and hopefully will stimulate further interdisciplinary thinking and studies in this exciting and growing area.

\section{AUTHOR CONTRIBUTIONS}

Drafted by SP. All authors contributed to the article and approved the submitted version.

\section{ACKNOWLEDGMENTS}

We thank the handling editors, Frontiers team and reviewers for their time, expertise and assistance in producing this special issue. 
Conflict of Interest: The authors declare that the research was conducted in the absence of any commercial or financial relationships that could be construed as a potential conflict of interest.

Publisher's Note: All claims expressed in this article are solely those of the authors and do not necessarily represent those of their affiliated organizations, or those of the publisher, the editors and the reviewers. Any product that may be evaluated in this article, or claim that may be made by its manufacturer, is not guaranteed or endorsed by the publisher.

Copyright (c) 2022 Pooley, Linnell, Münster, van Dooren and Zimmermann. This is an open-access article distributed under the terms of the Creative Commons Attribution License (CC BY). The use, distribution or reproduction in other forums is permitted, provided the original author(s) and the copyright owner(s) are credited and that the original publication in this journal is cited, in accordance with accepted academic practice. No use, distribution or reproduction is permitted which does not comply with these terms. 